statements of case-law signal a step towards formulaic, boilerplate text. Since, say, a decade, it seems that standardized pieces of text are fed into decisions whenever they fit - often just approximately. Pursuing other goals, this book obviously does not provide compelling proof of the verity of that second perspective. It can only be stated that the way the three interpretive formulas evolved in the last ten to twenty years reveals clear marks of boilerplate statements. Sometimes the use of standardized formulas seems unreflected in the light of the thrust and the outcome of the decision concerned. The feeling is hard to dispel that we have already moved quite deeply into legal automation.

\title{
II Breadth
}

In the free movement of persons and services the Court interprets certain notions broadly, while others restrictively. It also applies certain inherently expansive concepts. This is the story of the first interpretive formula which is narrated in this book. In truth we are looking at a set of interpretive formulas, encompassing in a first branch, inter alia, the early broad notion of 'worker', the later 'very broad' notion of establishment, and a broad notion of free movement in general; in a second branch narrow exceptions from rules; and in a third branch the apogee of broad interpretation, the 'greatest possible freedom'. These three branches can be clearly distinguished, but sometimes they 'touch' each other in a specific decision. Only the 'greatest possible freedom' has been confined to social security decisions, at least until very recently.

While the occurrences and the development of formulas of broad interpretation through the three branches and the impact they have can be read up in detail in the second part of this book - especially the crystal clear 'spin' and power of 'the greatest possible freedom' in a series of cases - a more general pattern of evolution in the case-law deserves mention. Early on, the Court of Justice posited a broad notion of 'worker' backed up soon by a restrictive reading of derogations from the free movement of workers. 'Worker' broadly understood subsequently evolved into a broad understanding of the free movement of workers more generally, an evolution that was largely complete by the time the Maastricht treaty came around. This might not be terribly surprising and may all be well known (though solid evidence for well-known facts is, of course, still scientifically valuable). Yet what is interesting is that this evolution was eventually mirrored for the freedom of services, once the services case-law took off. The evolution went from restrictive exceptions to the freedom of services over the broadly interpreted scope of the freedom to broadly construed provisions enshrining the free movement of services in general. In addition, a similar evolution can later be witnessed for Union citizenship. The Court began with some broad language both for workers and citizens, underpinned by a particularly restrictive interpretation of derogations from the freedom of workers, given Union citizen- 
ship. Soon the sweeping ruling was added that secondary legislation implementing citizenship in general was worthy of broad interpretation and the narrow understanding of restrictions of the freedom of citizens was fed in.

Relatively speaking, the freedom of establishment was held back in this regard, at least to some extent. The Court seemed to embark on a new course in terms of broad interpretation with the 'very broad' notion of establishment after Maastricht - this being one of the lesser well known aspects of Gebhard, 1995 but the interpretive evolution stopped there. The 'very broad' notion did not morph into a broad freedom of establishment in general, nor was it backed up by a narrow understanding of restrictions. It is possible though that the corresponding developments in the free movement of services cover the freedom of establishment, too, since the two freedoms are strongly entwined. Yet no clear evidence has emerged in this regard.

Possibly, the above pattern of evolution, which is now established clearly for the freedoms of workers, services, and Union citizens, is a mere technicality at risk of being over-interpreted. But a broader point should stick: 'broad notions' sometimes herald a more general expansive approach. Hence, scholars and practitioners should be alert when the Court sorts a seemingly innocent broad notion.

\section{Coordination}

In contrast to the frequent, manifold, and relatively complex occurrences of broad and restrictive interpretation, interpretation on the basis of 'mere coordination' is quite straightforward. Again, the details of the formula and its 'spin' can be studied in the relevant chapter, but certain points deserve to be mentioned here. Although other instruments also coordinate national law within the free movement of persons and services, 'mere coordination' is employed as an interpretive formula, as an argument with a certain impact in decisions, only in social security. In the coordination of social security 'mere coordination', however, performed a surprising and noteworthy volte-face. Initially, under the reign of Regulation 3, the idea that the national social security systems were 'merely coordinated' served as a justification for the Court to allow migrant workers to retain certain advantages. With the advent of the successor, Regulation 1408/71, which remained coordinative save in certain clearly circumscribed situations, the function of 'mere coordination' changed. In the subsequent decisions applying Regulation 1408/71 'mere coordination' served as an excuse for certain disadvantages migrant workers suffered. This reversal becomes very clear in Cabras, 1990, in particular when the case is contrasted to Keller, 1971 and Mancuso, 1973. The formula's volte-face is truly remarkable given that both Regulations 3 and 1408/71 rested on the idea of coordination rather than harmonization of na- 